\title{
Carbon nanotube-incorporated collagen hydrogels improve cell alignment and the performance of cardiac constructs
}

This article was published in the following Dove Press journal:

International Journal of Nanomedicine

13 April 2017

Number of times this article has been viewed

\author{
Hongyu Sun* \\ Jing Zhou* \\ Zhu Huang* \\ Linlin Qu* \\ Ning Lin* \\ Chengxiao Liang \\ Ruiwu Dai \\ Lijun Tang \\ Fuzhou Tian \\ General Surgery Center, Chengdu \\ Military General Hospital, Chengdu, \\ China \\ *These authors contributed equally \\ to this work
}

\begin{abstract}
Carbon nanotubes (CNTs) provide an essential 2-D microenvironment for cardiomyocyte growth and function. However, it remains to be elucidated whether CNT nanostructures can promote cell-cell integrity and facilitate the formation of functional tissues in 3-D hydrogels. Here, single-walled CNTs were incorporated into collagen hydrogels to fabricate (CNT/Col) hydrogels, which improved mechanical and electrical properties. The incorporation of CNTs (up to $1 \mathrm{wt} \%$ ) exhibited no toxicity to cardiomyocytes and enhanced cell adhesion and elongation. Through the use of immunohistochemical staining, transmission electron microscopy, and intracellular calcium-transient measurement, the incorporation of CNTs was found to improve cell alignment and assembly remarkably, which led to the formation of engineered cardiac tissues with stronger contraction potential. Importantly, cardiac tissues based on CNT/Col hydrogels were noted to have better functionality. Collectively, the incorporation of CNTs into the Col hydrogels improved cell alignment and the performance of cardiac constructs. Our study suggests that $\mathrm{CNT} / \mathrm{Col}$ hydrogels offer a promising tissue scaffold for cardiac constructs, and might serve as injectable biomaterials to deliver cell or drug molecules for cardiac regeneration following myocardial infarction in the near future.
\end{abstract}

Keywords: carbon nanotubes, collagen hydrogel, cardiac constructs, cell alignment, tissue functionality

\section{Introduction}

Cardiovascular diseases are the leading cause of death worldwide, especially myocardial infarction (MI). MI is a significant type among these diseases, and leads to a loss of myocardium impairment and scar-tissue formation, eventually resulting in heart failure. ${ }^{1,2}$ To date, various strategies have been developed to replace the scar tissue and restore cardiac functions..$^{3-6}$ However, these strategies have shown limited therapeutic efficacy in repairing the damaged myocardium. ${ }^{5,6}$

Cardiac tissue engineering offers a promising strategy for myocardial regeneration. The strategy is to seed specific cells within 3-D biomaterials to reconstruct their organization into contracting cardiac tissues, later to be transplanted to damaged myocardium to restore function. ${ }^{7-9}$ In order to realize successful cell assembly and efficient reconstitution, the biomaterial scaffold as the support for cell growth should allow effective integrity among cardiomyocytes and guide the formation of well-organized sarcomeric structures to contract efficiently. ${ }^{10}$ Recently, carbon nanotubes (CNTs), among the major promising nanomaterials applied in the biomedical field, have been reported to enhance the assembly of cultured cardiomyocytes in 2-D conditions. ${ }^{11-13}$ CNTs have incorporated into collagen (Col) substrates to fabricate nanocomposite
Correspondence: Fuzhou Tian; Lijun Tang Chengdu Military General Hospital, 270 Rongdu Avenue, Tianhui Road, Chengdu, Sichuan 610083, China

Tel/fax +86288657 I25।

Email tfz6689@I63.com; tanglj2016@163.com
International Journal of Nanomedicine 2017:12 3/09-3/20

3109 
matrices, in which cardiomyocytes showed homogeneous alignment, elongated sarcomeric structures along the longitudinal cell axis, and better synchronous contraction. ${ }^{13}$ These data suggest that CNTs provide an essential 2-D microenvironment for cardiomyocyte growth. However, it remains to be elucidated whether CNT nanostructures can promote cell-cell integrity and facilitate the formation of functional tissues in the 3-D microenvironment.

Among 3-D biomimetic scaffolds, hydrogels are used extensively in tissue engineering, due to their porous and hydrated molecular structure. ${ }^{14,15}$ Of the hydrogels available now, Col hydrogels have attracted the greatest interest. As the most abundant constituent of natural extracellular matrices of cardiac tissues, Col hydrogels have been utilized for cardiac tissue engineering. ${ }^{16,17}$ However, Col hydrogels are mechanically weak and electrically insulated, which leads to poor cell-cell integrity among cardiomyocytes and thereby diminishes functionality of the constructed tissues. More recently, the inclusion of electrically conductive nanomaterials has been demonstrated to address the current shortcomings of these conventional hydrogels. ${ }^{11,18,19}$ Specifically, CNTs, due to their outstanding mechanical and electrical properties, have been incorporated with Col to fabricate electroactive nanocomposite hydrogels. ${ }^{20,21}$ The obtained CNT/Col hydrogels have been investigated in the field of neural tissue engineering. For example, Tosun and McFetridge demonstrated that CNT/Col hydrogels exhibited enhanced mechanical and electrical properties and possessed positive biocompatibility of neuron-like cells in vitro. ${ }^{20}$ Inclusion of electrically conductive CNTs was also shown to increase neurite outgrowth within the Col hydrogels by Koppes et al. ${ }^{21}$ Although there has been progress made in neural tissue engineering, it remains to be seen whether $\mathrm{CNT} / \mathrm{Col}$ hydrogels exert beneficial effects on cardiomyocyte growth and facilitate the formation of functional tissues.
Here, we aimed to extend our previous 2-D work to 3-D to evaluate the behavior and growth of cardiomyocytes within CNT/Col hydrogels. Therefore, we utilized CNT/Col hydrogels and cardiomyocytes to construct cardiac tissues and compared these with Col hydrogels. We determined whether CNT/Col hydrogels can promote efficient integrity among cardiomyocytes and thus facilitate the formation of functional tissues in 3-D nanoscale (Figure 1). We found that the incorporation of CNTs within Col hydrogels enhanced cell adhesion and elongation and alignment of cardiomyocytes, leading to the formation of engineered cardiac tissues with stronger contraction potential.

\section{Materials and methods}

Sprague Dawley rats were purchased from Da Shuo Biotech Co Ltd (Chengdu, China). All experiments in this study were in compliance with the animal-care guidelines of the Ministry of Science and Technology of China and were approved by the animal experiments ethics committee of Chengdu Military General Hospital, Chengdu, China. We confirm that ethical and legal approval was obtained prior to the commencement of our study.

\section{Preparation of CNT/Col nanocomposite hydrogels}

$\mathrm{CNT} / \mathrm{Col}$ nanocomposite hydrogels were fabricated as previously described. ${ }^{20}$ In brief, carboxylic single-walled CNTs ( $>95 \%$ purity, $0.8-1.6 \mathrm{~nm}$ in diameter, and 5-30 $\mu \mathrm{m}$ in length) were purchased from Nanostructured and Amorphous Materials, Inc. (Houston, TX, USA). These were added into the solution type I Col from rat tails $(2.4 \mathrm{mg} / \mathrm{mL}$ in acetic acid) at varying CNT concentrations $(0,0.5,1$, and $2 \mathrm{wt} \% \mathrm{CNT}$ with respect to $\mathrm{Col})$. Subsequently, these mixture solutions were homogenized in an ultrasonic bath for 30 minutes. To obtain the composite hydrogels,

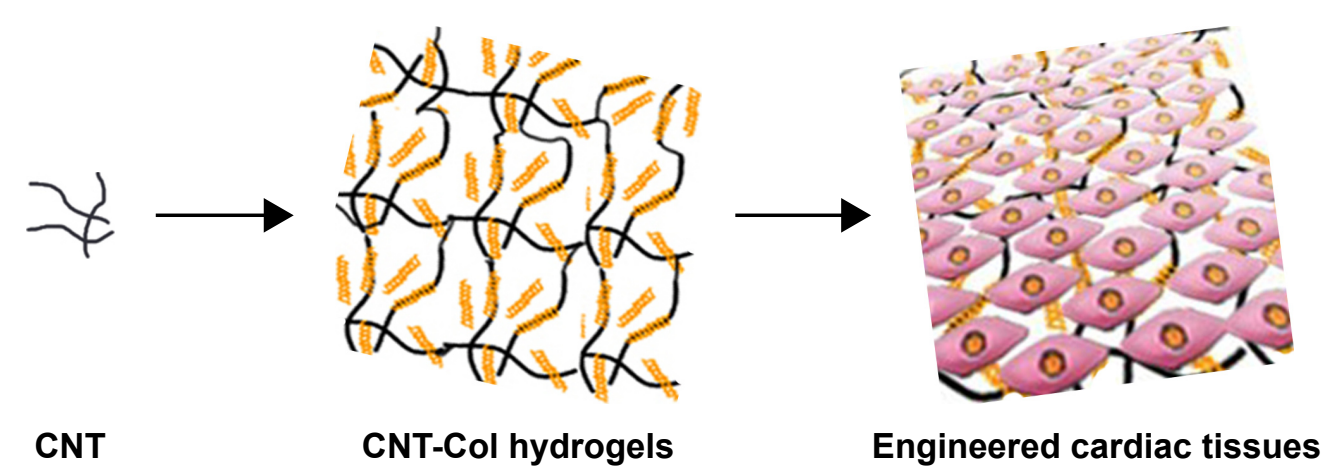

Figure I Schematic representation to describe the study.

Note: CNT/Col hydrogels promoted efficient integrity among cardiomyocytes, and thus facilitated the formation of functional tissues in 3-D nanoscale cues. Abbreviations: CNT, carbon nanotube; Col, collagen. 
homogeneous solutions, double-concentrated Dulbecco's Modified Eagle's Medium- $\alpha$, and fetal bovine serum were mixed 4:5:1 (v/v) on ice, poured into 24-well tissue-culture plates, and then incubated at $37^{\circ} \mathrm{C}$ to allow gelation within 15 minutes. For the preparation of $\mathrm{Col}$ hydrogels, an appropriate amount of $1 \mathrm{M} \mathrm{NaOH}$ solution was used to neutralize the Col mixture to $\mathrm{pH} 7.4$, and then placed in a humidified incubator to polymerize at $37^{\circ} \mathrm{C}$.

\section{Characterization of CNT/Col nanocomposite hydrogels}

Scanning electron microscopy (S-3400N; Hitachi, Tokyo, Japan) was used to observe the microstructure of CNTs embedded within the CNT/Col hydrogels. After freezedrying, the samples were sputter-coated with gold (Autoconductavac IV, See-Vac) and imaged with microscopy under a working voltage of $15 \mathrm{kV}$.

The mechanical properties of the hydrogels were assessed by a TA.XT2 $\mathrm{i}$ texture analyzer (Texture Technologies, Hamilton, MA, USA). The hydrogel samples were measured from the slope in the linear region corresponding to $5 \%-15 \%$ strain at $0.1 \mathrm{~mm} /$ second. Obtained compressive modulus values were averaged. Experiments were conducted in triplicate for each group.

The conductivity of the hydrogels was assessed by a three-point probe (Oakton Instruments, Vernon Hills, IL, USA). The hydrogels were submerged in saline solution, and four conductivity readings per sample were acquired at a frequency of $27 \mathrm{kHz}$ for each hydrogel.

\section{Construction of cardiac tissue based on CNT/Col hydrogels}

Neonatal rat ventricular myocytes (NRVMs) were isolated from 1-day-old neonatal Sprague Dawley rats as previously described. ${ }^{13}$ At the gelation stage of the hydrogels, NRVMs were embedded within CNT/Col or Col hydrogels using Dulbecco's Modified Eagle's Medium with the suspension $\left(2 \times 10^{6}\right.$ cells $\left./ \mathrm{mL}\right)$. Subsequently, CNT/Col- or Col hydrogelbased constructs were cultured in normal medium at $37^{\circ} \mathrm{C}$ in an atmosphere of $5 \% \mathrm{CO}_{2}$. The medium was changed every other day until sampling.

\section{Cell-viability assays}

The effect of CNTs on cell viability in the cell--hydrogel constructs was assessed by live/dead staining and AlamarBlue assays. A live/dead viability/cytotoxicity kit (Thermo Fisher Scientific, Waltham, MA, USA) was utilized to measure the viability of NRVMs within CNT/Col and Col hydrogels.
Over 1- and 3-day cultivation periods, cell-hydrogel constructs were incubated for approximately 10 minutes with $2 \mu \mathrm{M}$ calcein acetoxymethyl (AM) and $4 \mu \mathrm{M}$ ethidium homodimer 1 in phosphate-buffered saline (PBS). Ten randomly selected fields were imaged with fluorescence microscopy (AZ-100 multipurpose microscope; Nikon, Tokyo, Japan). The images obtained were processed by ImageJ software (National Institutes of Health; NIH, Bethesda, MD, USA). In addition, AlamarBlue-based colorimetric assays were carried out, in which cytotoxicity evaluation was based on metabolic activity. Independent experiments were conducted three times for each group.

\section{Measurement of cell adhesion and elongation}

The effect of CNTs on the morphological variation of NRVMs within the hydrogels was determined by fluorescence staining of F-actin. In brief, after 1 and 2 days of culture, cellhydrogel constructs were fixed with $4 \%$ paraformaldehyde and then stained with fluorescein isothiocyanate-conjugated phalloidin (Thermo Fisher Scientific). Cell nuclei were stained with 4',6-diamidino-2-phenylindole (DAPI) (Thermo Fisher Scientific). Ten pictures per section under 20× fields of NRVMs within CNT/Col or Col hydrogels were randomly imaged by confocal microscopy and analyzed using Image J software. In addition, cell area and long/short axis of NRVMs at day 1 and 2 were analyzed with ImageJ software. An average of 200 cells was counted for each sample group. After 7 days of culture, cell-hydrogel constructs were fixed in $4 \%$ formaldehyde in PBS and stained with hematoxylin and eosin for general evaluation.

\section{Immunofluorescence staining and confocal microscopy}

For immunofluorescence staining, the cell-hydrogel constructs were fixed in $4 \%$ formaldehyde for 30 minutes, followed by treatment with $0.3 \%$ Triton X-100 in PBS for 10 minutes. Then, the samples were blocked in $2 \%$ bovine serum albumin for 45 minutes to block nonspecific antibody binding. Subsequently, the samples were incubated with the primary antibodies: mouse monoclonal anti- $\alpha$-sarcomeric actinin ( $\alpha \mathrm{SA} ; 1: 100$; Abcam, Cambridge, UK), rabbit monoclonal anti-troponin I (TnI; 1:100; Abcam), and rabbit monoclonal anti-Cx43 (1:500; Abcam) overnight at $4^{\circ} \mathrm{C}$. The samples were then washed three times in PBS and stained with Alexa Fluor 488-conjugated secondary antibodies (1:500; Thermo Fisher Scientific). Images were acquired by a Zeiss LSM 510 microscope. Sarcomere length and Z-line 
width were assessed using Image J software, in accordance with previous reports. ${ }^{22,23}$ Ten $\alpha \mathrm{SA}^{+}$cells were measured, with ten measurements for each cell.

\section{Transmission electron microscopy (TEM) analysis of cell-hydrogel constructs}

Cell-hydrogel constructs were fixed in $2 \%$ paraformaldehyde $/ 2.5 \%$ glutaraldehyde and postfixed in $1 \%$ phosphatebuffered $\mathrm{OsO}_{4}$ for 2 hours. After dehydration, the samples were embedded in epoxy resin, and semithin sections were stained with toluidine blue. Samples were imaged using TEM (Tecnai 10; Thermo Fisher Scientific).

\section{Analysis of beating behavior of cells}

The spontaneous beating behavior of NRVMs was determined after 7 days of culture. Representative beating behaviors were captured from cell-hydrogel constructs, and each group was six replicates of the cell-hydrogel constructs.

\section{Intracellular calcium-transient measurements}

After 7 days of the culture, cell-hydrogel constructs were loaded with $10 \mathrm{mM}$ Fluo-4 AM (Thermo Fisher Scientific) and 0.1\% Pluronic F127 (Sigma-Aldrich, St Louis, MO, USA) in Tyrode's solution for 45 minutes at $37^{\circ} \mathrm{C}$. Next, the samples were soaked in normal Tyrode's solution to allow de-esterification. The spontaneous intracellular $\mathrm{Ca}^{2+}$ transient was imaged using confocal microscopy at excitation wavelengths of $488 \mathrm{~nm}$. Fluorescence (F) during cells contractions was normalized to the basal cell fluorescence after dye loading $\left(\mathrm{F}_{0}\right)$. Volocity software was utilized to analyze the images. Intracellular $\mathrm{Ca}^{2+}$ was presented as:

$$
\begin{aligned}
{\left[\mathrm{Ca}^{2^{+}}\right]_{\mathrm{i}} } & =\mathrm{Kd}\left(\mathrm{F} / \mathrm{F}_{0}\right) /\left(\mathrm{Kd} /[\mathrm{Ca}]_{\mathrm{i}} \text {-rest }+1-\mathrm{F} / \mathrm{F}_{0}\right) \text { with } \mathrm{Kd} \\
& =1,100 \mathrm{nmol} / \mathrm{L} \text { and }\left[\mathrm{Ca}^{2^{+}}\right]_{\mathrm{i}}-\text { rest }=100 \mathrm{nmol} / \mathrm{L}
\end{aligned}
$$

\section{Statistical analysis}

All data are presented as mean \pm standard error of mean. Differences between two groups were assessed using the Student's unpaired two-tailed $t$-test. One-way analyses of variance with Tukey's post hoc tests were performed to analyze group differences. $P$-values $<0.05$ were considered statistically significant. All statistical tests were conducted with SAS statistical software version 9.1 (SAS Institute, Cary, NC, USA).

\section{Results \\ Physical characteristics of CNT/Col nanocomposite hydrogels}

Effects of CNT inclusion on morphological and structural properties of Col hydrogels can be seen in Figure 2. Ultrahigh-resolution scanning electron microscopy showed CNTs distributed on the fibrous structure of $\mathrm{Col}$ within the CNT/Col composite hydrogels (Figure 2A and B). As shown in Figure 2C, obvious increases in elastic moduli values were observed with the inclusion of CNTs embedded within the Col hydrogels. The elastic moduli values for $\mathrm{Col}$ and CNT/Col hydrogels at varying concentrations of CNTs from 0.5 to $2 \mathrm{wt} \%$ were $13.2 \pm 0.1,21.1 \pm 0.1,24.6 \pm 0.07$, and $28.8 \pm 0.092 \mathrm{kPa}$, similar to those of adult rat ventricular myocardium $(20 \pm 4-54 \pm 8 \mathrm{kPa}) .{ }^{24}$ Additionally, the inclusion of CNTs embedded within the Col hydrogels revealed a significant increase in conductivity compared with the pure Col hydrogels (Figure 2D). Moreover, the conductivity of CNT/Col hydrogels was slightly higher than that of native myocardium (ranging from 160 longitudinally to $5 \mathrm{mS} / \mathrm{m}$ transversely). ${ }^{25}$ These results indicated that CNT/Col hydrogel might be a promising material for engineering cardiac tissues.

\section{Cell viability and proliferation within the hydrogels}

To evaluate whether the inclusion of CNTs into hydrogels affected cell viability, NRVMs were seeded within CNT/Col hydrogels containing varying concentrations of CNTs $(0$, $0.5,1$, and $2 \mathrm{wt} \%$ ) to fabricate the cell-hydrogel cardiac constructs. According to the live/dead viability assay, NRVMs grown within $\mathrm{CNT} / \mathrm{Col}$ hydrogels containing CNTs up to $1 \mathrm{wt} \%$ displayed $>85 \%$ viability, which was similar to those within Col hydrogels (Figure 3A and B). However, cell viability was significantly decreased with concentrations of CNTs up to $2 \mathrm{wt} \%$, suggesting higher concentrations of CNTs incorporated within hydrogels had evident cell toxicity. Additionally, through the AlamarBlue-based assay (Figure 3C), no apparent changes in the relative number of live cells were noted among the four groups after culture for 24 hours, indicating that the incorporation of CNTs in hydrogels had no significant effect on the proliferation of NRVMs. Based on these results and the fact that electrical and mechanical properties of composite hydrogels were also positively correlated with CNT concentrations, we utilized Col hydrogels containing $1 \mathrm{wt} \%$ CNTs for further experiments. 
A

Col

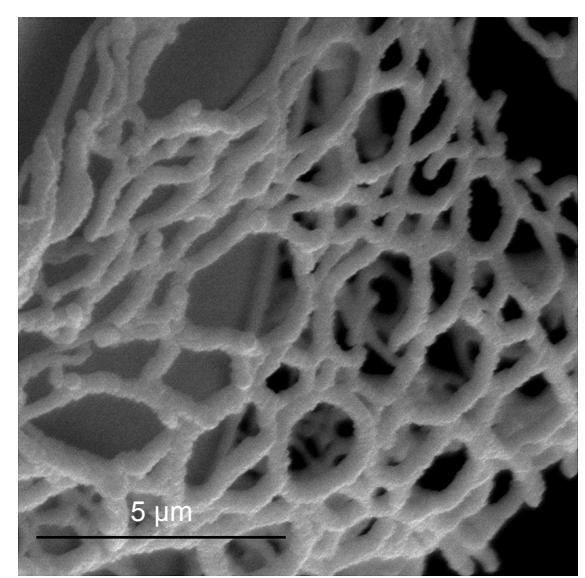

C

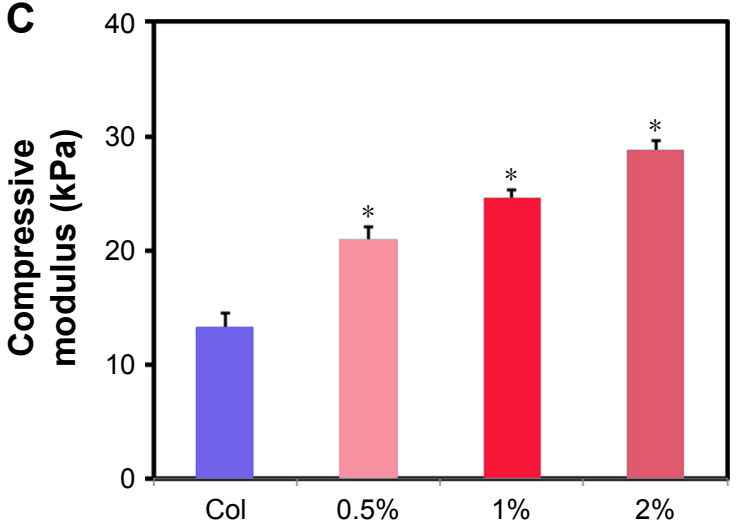

B
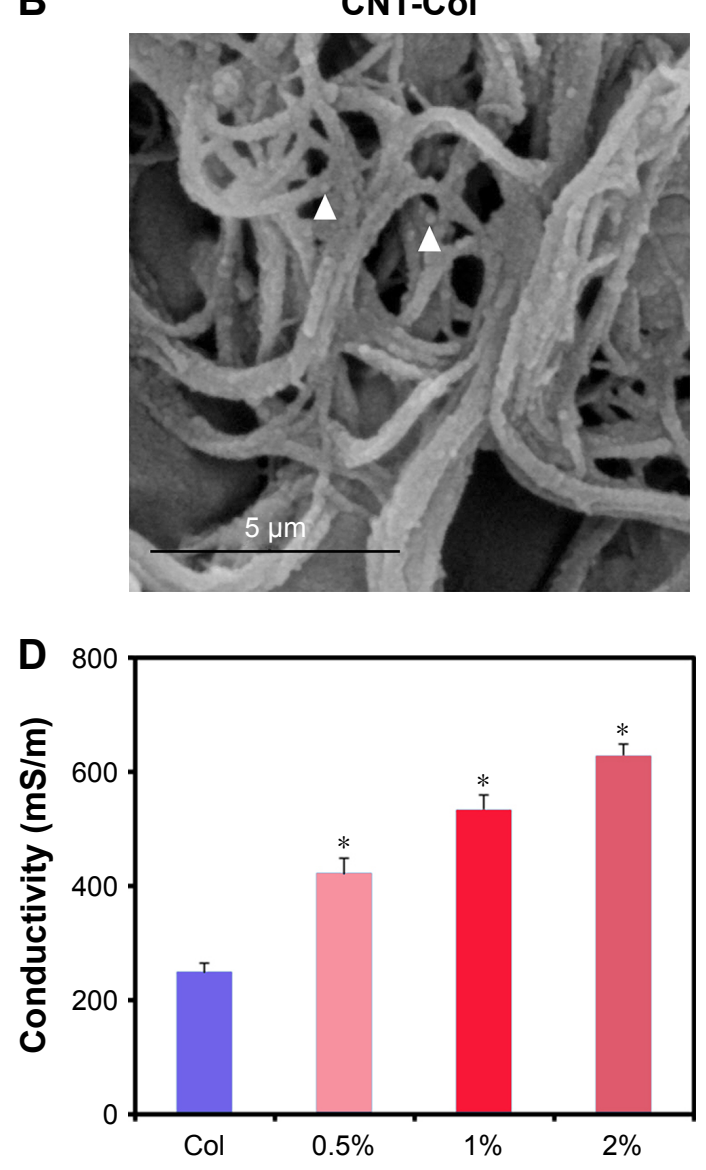

Figure 2 Physical characteristics of CNT/Col nanocomposite hydrogels.

Notes: (A, B) Ultrahigh-resolution scanning electron microscopy images show CNTs (white triangles) distributed on the fibrous structure of Col within the CNT/Col composite hydrogels. (C) Significant increases in elastic moduli values were observed in CNTs embedded within the Col hydrogels at varying concentrations of CNTs, from 0 to 2 wt\%. (D) The incorporation of CNTs within the Col hydrogels resulted in a significant increase in conductivity compared with the pure-Col hydrogels. Data are mean \pm standard error of mean. $* P<0.05$. All experiments were performed in triplicate.

Abbreviations: CNTs, carbon nanotubes; Col, collagen.

\section{Incorporation of CNTs within Col hydrogels promoted cell adhesion}

To evaluate the inclusion of CNTs into hydrogels on cell adhesion of NRVMs, we imaged the cells attaching to the hydrogel substrates using F-actin staining. As shown in Figure 4, structural changes in NRVMs were visualized clearly, due to the inclusion of CNTs within hydrogels on days 1 and 2. Remarkably, NRVMs within CNT/Col hydrogels exhibited more elongation and thicker actin filaments compared to those within Col hydrogels (Figure 4A). To quantify the correlation of CNTs and adhesion of NRVMs, we calculated the number of NRVMs per field, the area of individual NRVMs, and the long/short axis length by ImageJ software. As seen in Figure 4B and C, CNT/Col hydrogels significantly increased the number of adherent cells and the adherent area of individual NRVMs compared with the Col group. Meanwhile, the ratios of long and short axial lengths of the NRVMs on CNT/Col hydrogels were smaller than those on Col hydrogels (Figure 4D). These results indicated that the inclusion of CNTs within Col hydrogels promoted cell adhesion and elongation.

\section{Incorporation of CNTs promoted cell alignment and assembly in cardiac constructs}

To evaluate the effects of CNTs on the phenotype of cardiac constructs, the morphology of NRVMs was observed by phasecontrast microscopy. As seen in Figure 5A, NRVMs mainly displayed a round shape within Col hydrogels, while apparently stretching cells were noted within CNT/Col hydrogels at day 7. Hematoxylin and eosin staining (Figure 5B) showed that NRVMs grown within the CNT/Col hydrogels formed well-organized tissues, in which NRVMs exhibited elongated and aligned morphology, and possessed elongated nuclei. 
A

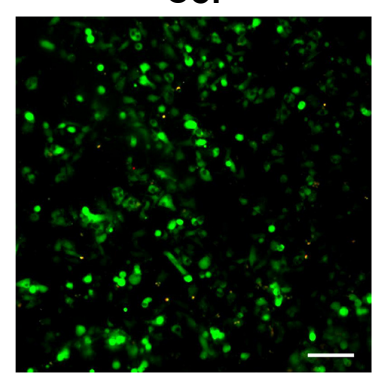

B

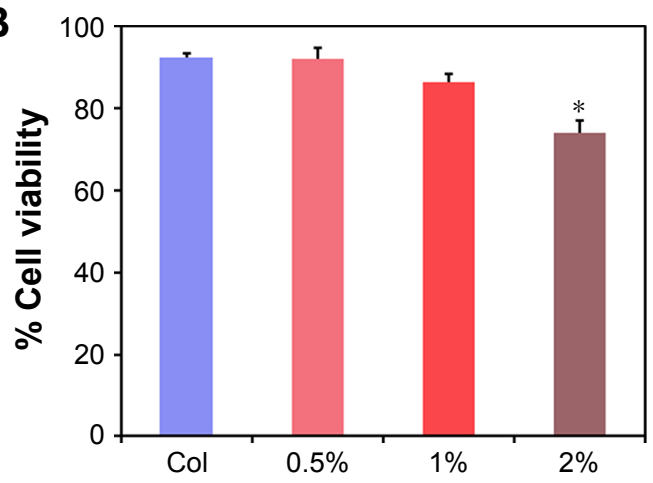

$0.5 \%$ CNT

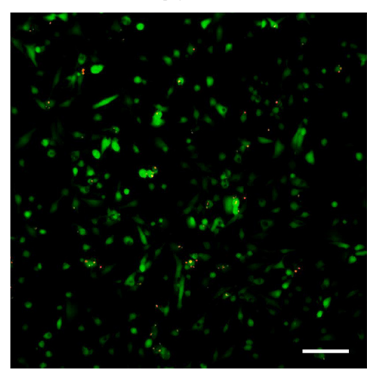

$1 \%$ CNT

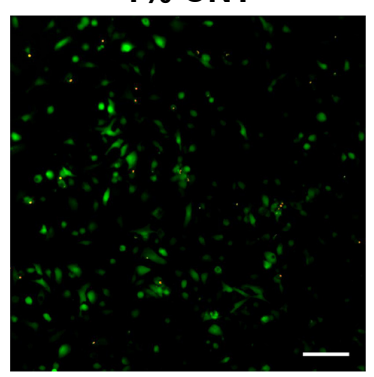

$2 \%$ CNT
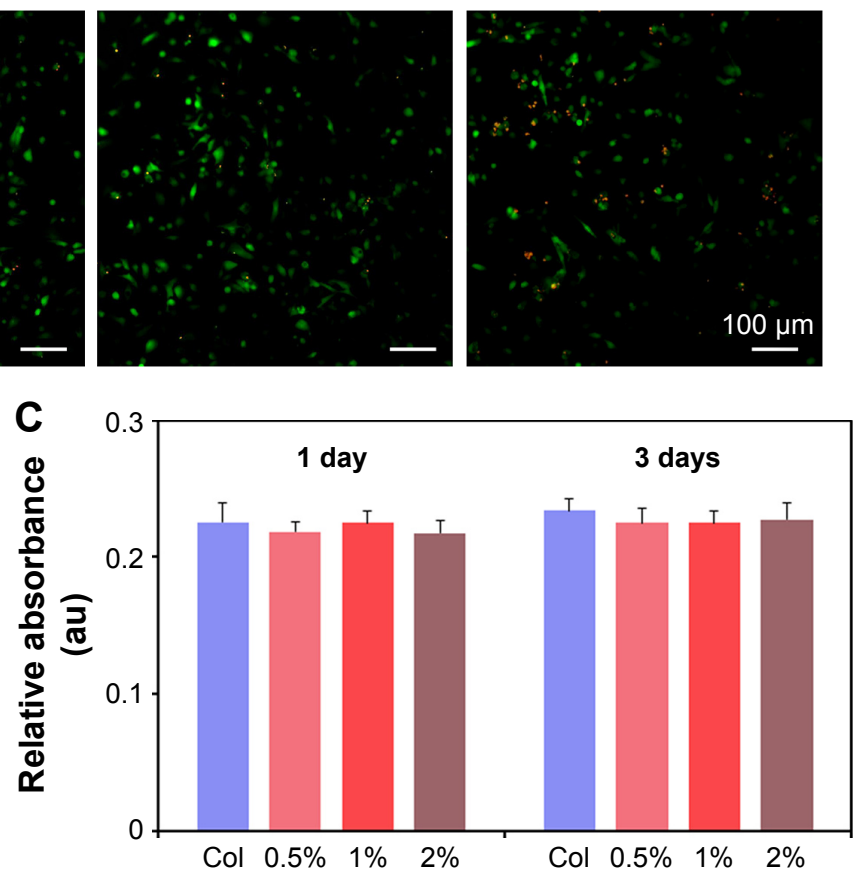

Figure 3 Cell viability and proliferation within the hydrogels.

Notes: (A, B) Live/dead assays showing cell viability of NRVMs within CNT/Col hydrogels containing varying concentrations of CNTs (0, 0.5 , I, and 2 wt\%) at day 3. (C) AlamarBlue viability assay showed no apparent changes in relative number of live cells among the four groups after culture at days I and 3. Data are mean \pm standard error of mean. $* P<0.05$. All experiments were performed in triplicate.

Abbreviations: NRVMs, neonatal rat ventricular myocytes; CNT, carbon nanotube; Col, collagen.

A

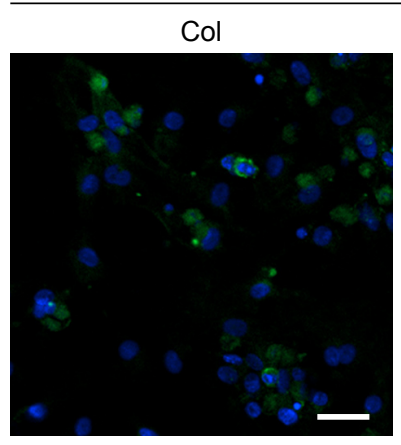

B

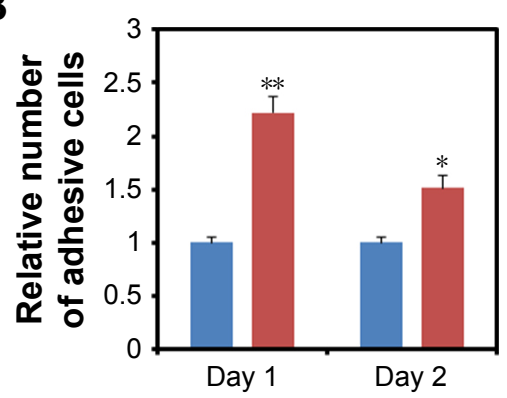

Day 1

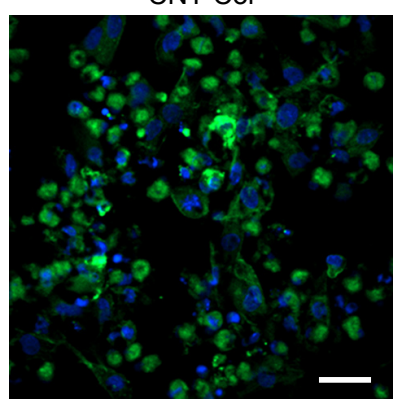

C

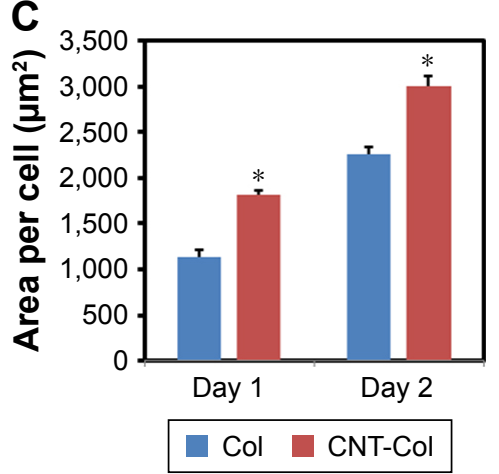

Day 2
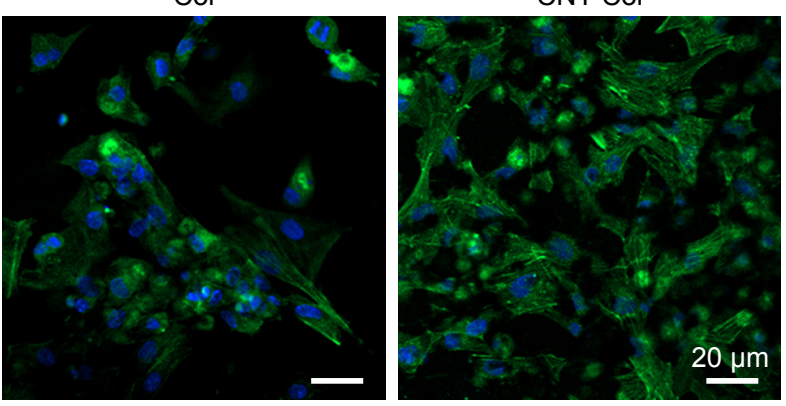

D

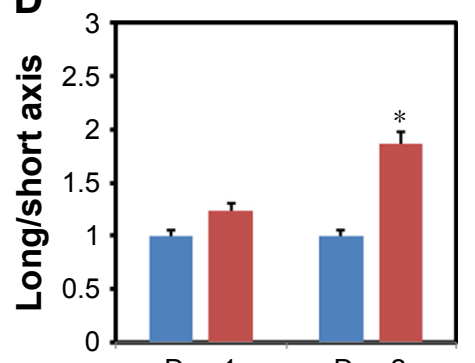

Day 1

Figure 4 Incorporation of CNTs within Col hydrogels promoted cell adhesion.

Notes: (A) Fluorescence staining of F-actin showed that NRVMs within CNT/Col hydrogels exhibited more elongation and thicker actin filaments compared to those within Col hydrogels. (B-D) Percentages of cell adhesion (B), area of individual cells (C), and long/short axis (D) within CNT/Col hydrogels were significantly higher than those on Col hydrogels at days I and 2. Data are mean \pm standard error of mean. $* P<0.05 ; * P<0.01$. All experiments were performed in triplicate.

Abbreviations: CNTs, carbon nanotubes; Col, collagen; NRVMs, neonatal rat ventricular myocytes. 

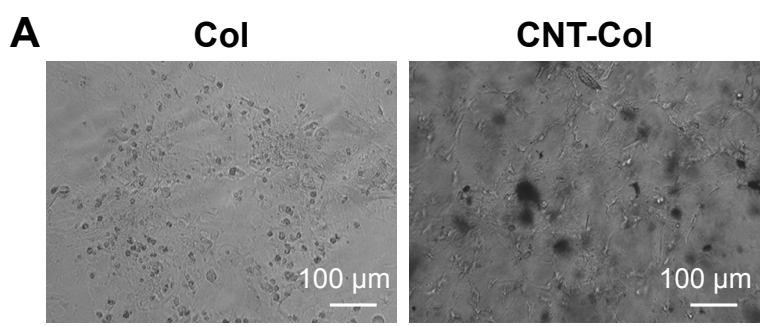

B
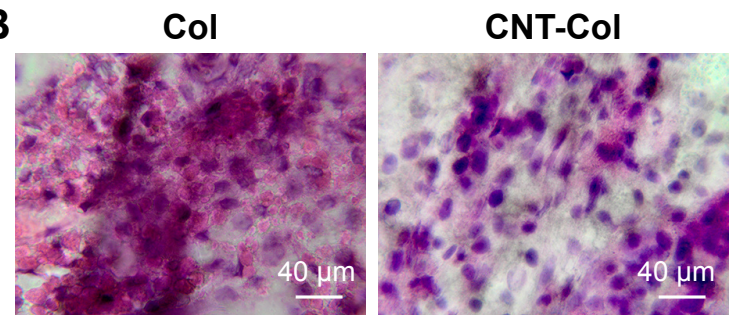

C

$\alpha S A$
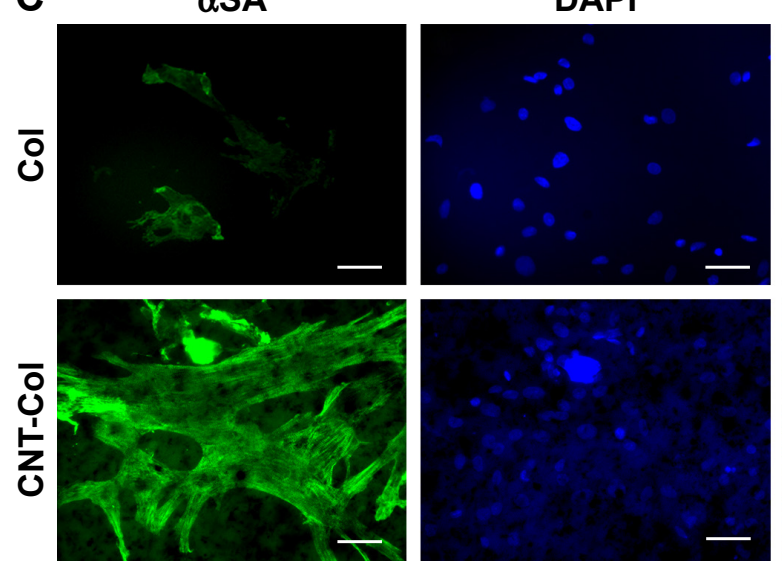

D

Tnl
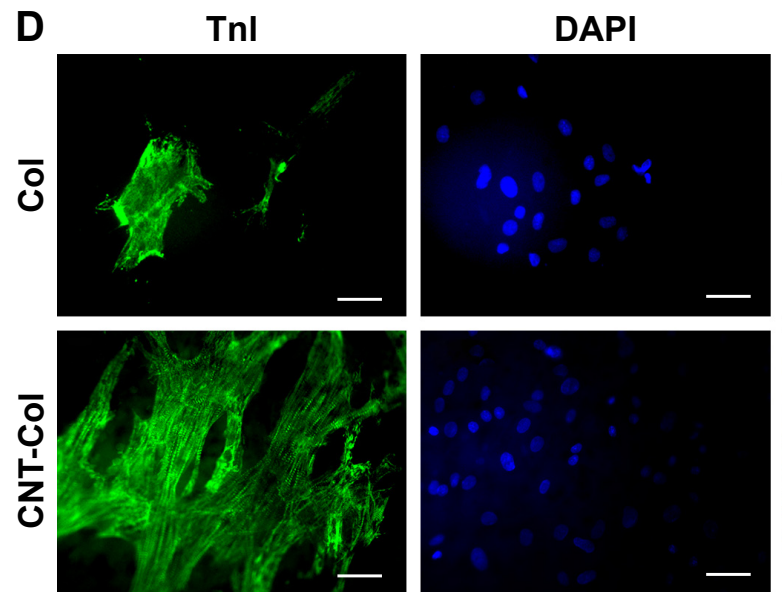

Merged

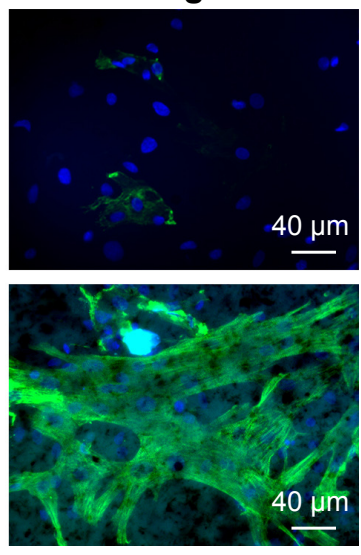

Merged
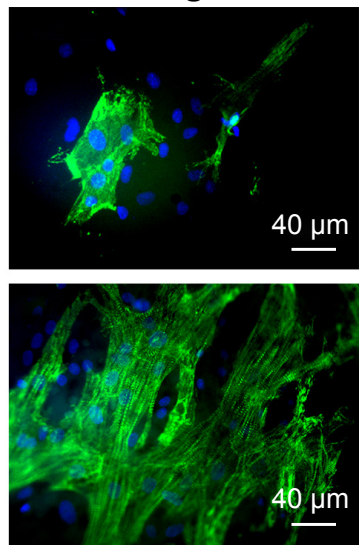

Figure 5 Incorporation of CNTs improved cell alignment and assembly in cardiac constructs.

Notes: (A) Optical images of NRVMs revealed better cell stretch and more homogeneous distribution within CNT/Col than Col hydrogels at day 7. (B) Hematoxylin and eosin staining showed that NRVMs grown within the CNT/Col hydrogels formed well-organized tissues, while NRVMs grown within Col hydrogels displayed random alignment with certain aggregation. (C, D) Immunostaining of $\alpha$-sarcomeric actin ( $\alpha$ SA, green) and troponin I (Tnl; green) revealed that NRVMs within CNT/Col hydrogels exhibited much more aligned and elongated morphology with massive actinin striation compared to the control group on day 7.

Abbreviations: CNTs, carbon nanotubes; NRVMs, neonatal rat ventricular myocytes; Col, collagen.

In contrast, NRVMs grown within Col hydrogels displayed random alignment with some aggregation.

Next, we sought to assess the potential effects of CNTs to improve cardiomyocyte alignment and assembly grown within $\mathrm{CNT} / \mathrm{Col}$ hydrogels. After 7 days of culture, $\mathrm{CNT} / \mathrm{Col}$ - and Col hydrogel-based cardiac constructs were immunostained for $\alpha \mathrm{SA}$ and TnI, cardiac-specific markers associated with contraction. As shown in Figure 5C, NRVMs grown within $\mathrm{CNT} / \mathrm{Col}$ hydrogels exhibited aligned and elongated morphology with massive actinin striation. The NRVMs organized into elongated and aligned sarcomeric structures, resembling the cardiac cell bundles of the natural myocardium. However, the number of sarcomere bundles in NRVMs within Col hydrogels was lower relative to that within CNT/Col hydrogels. Similar observations were made in TnI immunostained images (Figure 5D), showing that cardiac constructs based on CNT/Col hydrogels exhibited a more extended architecture as a marker of contractile machinery compared with the control group.

Additionally, the gap-junction protein $\mathrm{Cx} 43$ was observed to localize in perinuclear regions and cell membranes for 7 days of cultivation in the Col group (Figure 6). 

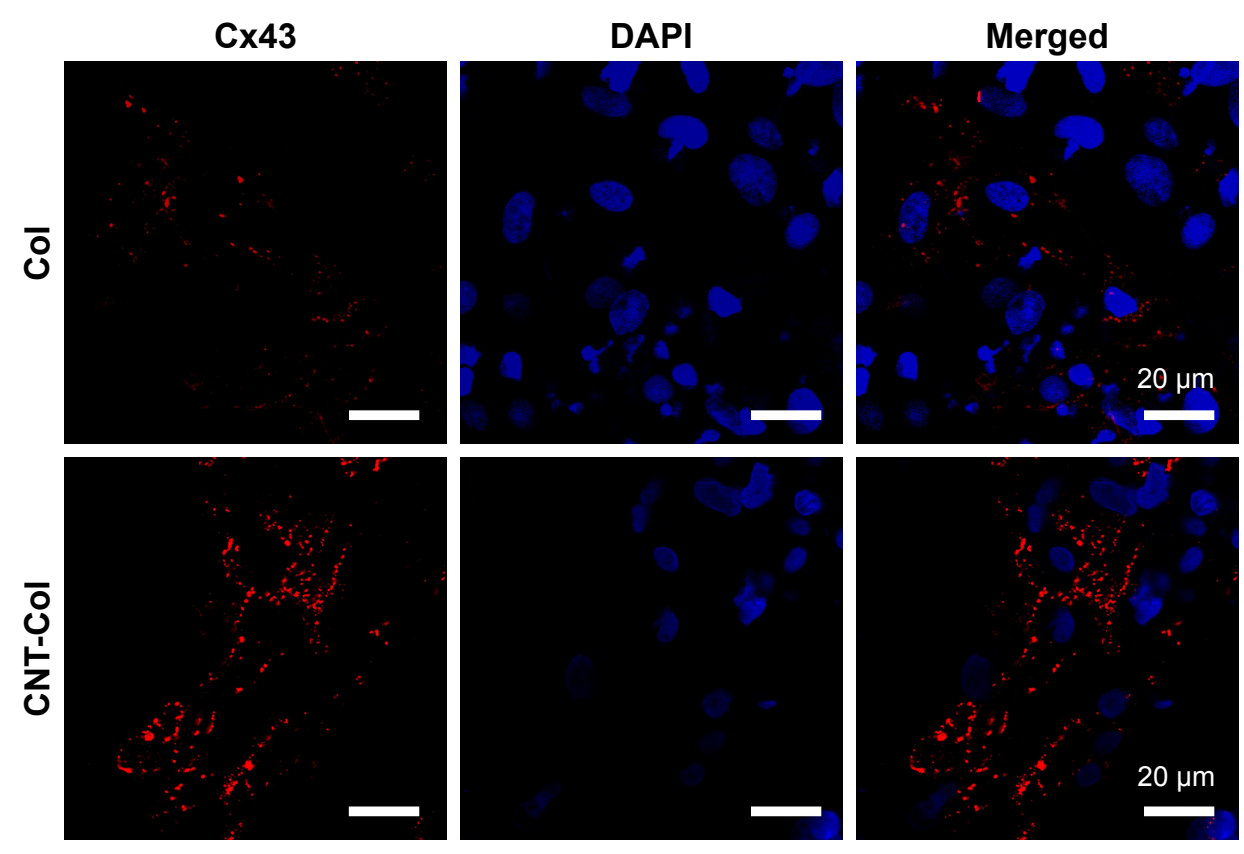

Figure 6 Immunofluorescent staining of Cx43 protein in cardiac constructs for 7 days.

Notes: Cx43 was observed to localize in perinuclear regions and cell membranes in the Col group. In contrast, within CNT/Col hydrogels, Cx43 was distributed in intercellular regions in either a punctate pattern or in linear plaques.

Abbreviations: Col, collagen; CNT, carbon nanotube.

In contrast, within CNT/Col hydrogels, $\mathrm{Cx} 43$ was distributed in intercellular regions in either a punctate pattern or in linear plaques. Compared to the control group, NRVMs within CNT/Col hydrogels exhibited obviously higher levels of $\mathrm{Cx} 43$ protein and had more numerous gap junctions, indicating that the inclusion of CNTs improved cardiomyocyte maturation. Overall, the inclusion of CNTs into the hydrogels improved cell alignment and assembly, leading to the formation of constructed cardiac tissue with better structure and phenotype.

\section{CNT/Col hydrogels enhanced cell assembly at ultramicrostructure level}

To evaluate further the effects of CNTs on the ultrastructural assembly of NRVMs, TEM of cell-hydrogel constructs was performed after culture of 3 and 7 days. As shown in Figure 7A, significantly longer and better-aligned registers of sarcomeric structures were observed in $\mathrm{CNT} / \mathrm{Col}$ hydrogels cardiac constructs than in the control group. The well-organized sarcomeres contained clearly visible I, Z (immature), and $\mathrm{H}$ bands (mature), which is the hallmark of native cardiac muscle cells. However, few sarcomeres were observed in Col hydrogels based cardiac constructs (Figure 7B). The data further indicated that the inclusion of CNTs enhanced cardiomyocyte assembly at an ultramicrostructure level.

\section{CNT/Col hydrogels enhanced the functionality of cardiac constructs}

To assess the impact of CNTs on tissue functionalities, we analyzed beating behavior of cell-hydrogel constructs. After culture of 3 days, NRVMs grown within CNT/Col hydrogels began to contract spontaneously in a synchronous manner. As shown in Figure 8A, a significant increase in beating frequency was observed in cardiac constructs based on CNT/Col hydrogels when compared to pure Col hydrogels after 7 days.

To examine further the capability of CNTs on tissue-level functionality, we performed calcium-transient measurements to measure intracellular $\mathrm{Ca}^{2+}$ concentration using Fluo-4 AM. As seen in Figure 8B-E, CNT/Col hydrogel-based cardiac constructs displayed apparently spontaneous relative synchronous $\mathrm{Ca}^{2+}$ transients and higher $\mathrm{Ca}^{2+}$ fluctuation in comparison to the control group. Furthermore, the average value of intracellular $\mathrm{Ca}^{2+}$ concentration in NRVMs $(251 \pm 21 \mathrm{nmol} / \mathrm{L}, \mathrm{n}=30)$ within $\mathrm{CNT} / \mathrm{Col}$ hydrogels significantly increased when compared to those $(193 \pm 11 \mathrm{nmol} / \mathrm{L}, \mathrm{n}=30)$ in Col hydrogels (Figure 8F).

\section{Discussion}

In this work, we extended our previous work and provided evidence that the incorporation of CNTs within Col hydrogels enhanced cell adhesion, elongation, and alignment of 

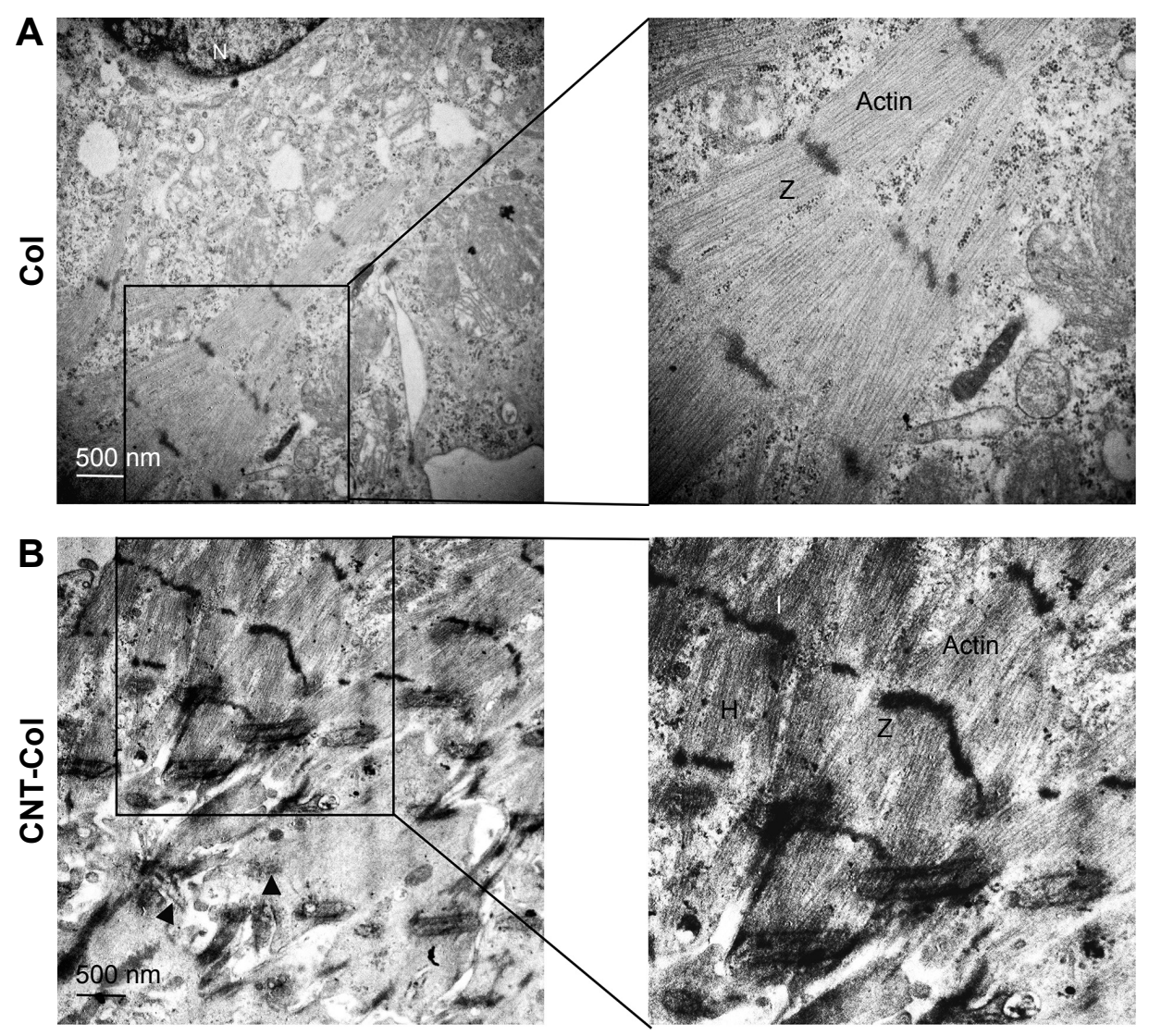

Figure 7 Ultramicrostructures and sarcomeric structures in cardiac constructs for 7 days by electron microscopy.

Notes: (A) Few sarcomeres were observed in Col hydrogel-based cardiac constructs. (B) Significantly longer and better-aligned registers of sarcomeric structures were observed in CNT/Col hydrogel cardiac constructs than in the control group. N, nucleus; Z, Z-line; I, I band; H, H band; black triangles, CNTs.

Abbreviations: Col, collagen; CNT, carbon nanotube.

cardiomyocytes, leading to the formation of engineered cardiac tissues with stronger contraction potential. These results suggest that $\mathrm{CNT} / \mathrm{Col}$ hydrogels offer a promising tissue scaffold for cardiac constructs, and may serve as injectable biomaterials to deliver cell or drug molecules for cardiac regeneration following $\mathrm{MI}$ in the near future.

Due to their inertness and hydrophobicity, native CNTs are difficult to dissolve in aqueous solutions, thereby hampering homogeneous dispersion and ultimately leading to significant toxicity. ${ }^{26,27}$ To avoid this weakness, CNTs are often functionalized by modifying their surface chemistry, and functionalized CNTs have been shown to affect cell behavior significantly. Therefore, we utilized carboxylic CNTs to fabricate 3-D hydrogel scaffolds, which allowed homogeneous mixing with I type Col solution and subsequent gelation of composite solutions containing CNTs. The obtained CNT/Col hydrogels (up to $1 \mathrm{wt} \%$ ) exhibited no significant cytotoxic effects on cardiomyocyte viability according to in vitro biocompatibility experiments. In agreement with our work, MacDonald et al reported fibroblast cells grown within CNT/Col-hydrogel matrices had high cell viability in the range of $80 \%-90 \% .{ }^{28}$ Our results were also consistent with recent work that $\mathrm{CNT} / \mathrm{Col}$ hydrogels with small concentrations of CNTs (up to $1 \mathrm{wt} \%$ ) were not toxic to mesenchymal stem cells. ${ }^{29}$

Col-hydrogel scaffolds are used extensively for cardiac tissue engineering. ${ }^{16,17}$ Although they have good biocompatibility, Col hydrogels display poor biomechanical properties and thus are limited in tissue-engineering applications. Due to their outstanding mechanical and electrical properties, CNTs have been utilized to incorporate into traditional scaffolds to improve the mechanical and electrical properties of the polymers, which emerge as promising nanomaterials in tissue-engineering research. ${ }^{11-14,20,21}$ To this end, CNTs were incorporated into a 3-D Col-hydrogel matrix to fabricate CNT/Col hydrogels, which have been used in neural tissue engineering. ${ }^{20,21,29}$ For example, Lee et al reported that $\mathrm{CNT} / \mathrm{Col}$ hydrogels had the ability to promote cell elongation and neural cell differentiation of mesenchymal stem cells. ${ }^{29}$ Additionally, CNT/Col hydrogels have also been applied in and cartilage tissue engineering. ${ }^{30,31}$ In the present study, we first applied CNT/Col hydrogels to cardiac tissue engineering, 
A

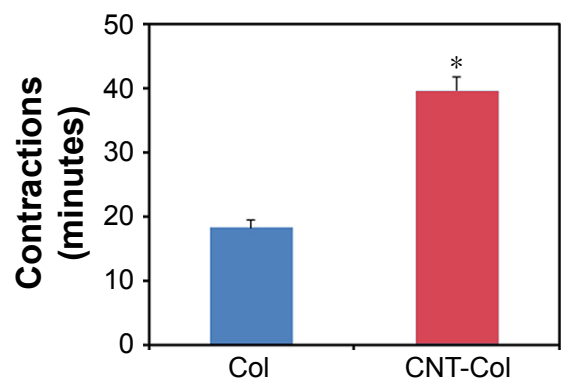

D

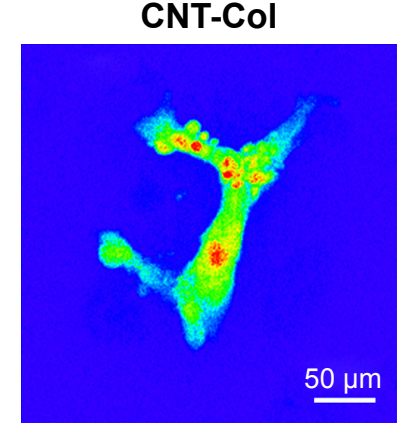

B

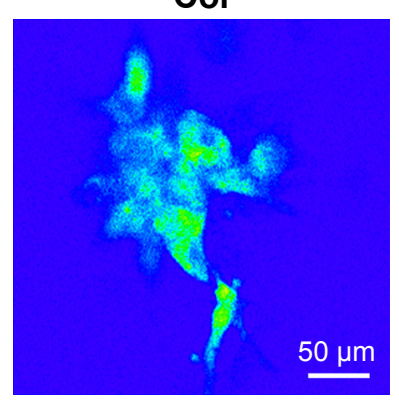

E

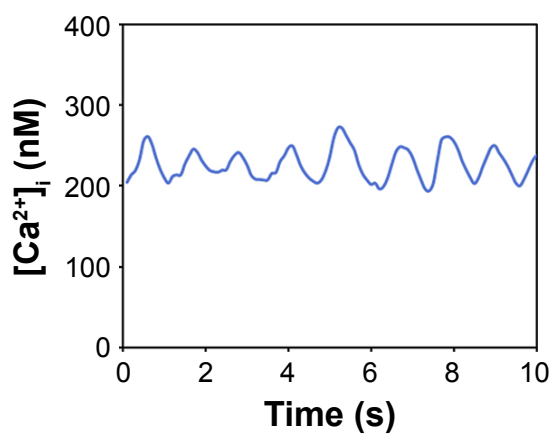

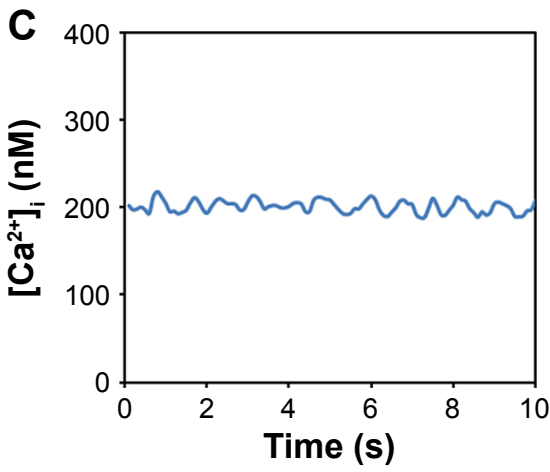

$\mathbf{F}$

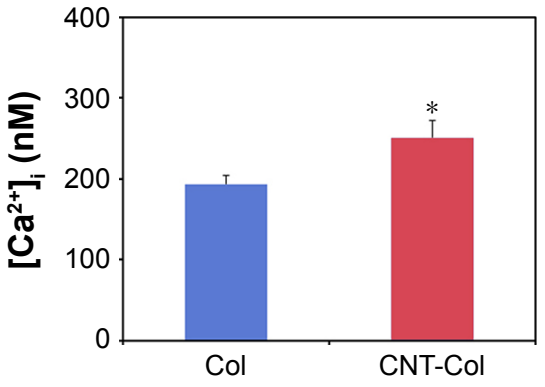

Figure 8 Incorporation of CNTs enhanced the functionality of cardiac constructs based on CNT/Col hydrogels.

Notes: (A) A significant increase in beating frequency was observed in cardiac constructs based on CNT/Col hydrogels when compared to pure Col hydrogels after 7 days. (B) Spontaneous $\mathrm{Ca}^{2+}$ transients in cardiac constructs within Col hydrogels on day 7. (C) Time-course of intracellular $\mathrm{Ca}^{2+}$-transient changes in the Col group. (D) Spontaneous $\mathrm{Ca}^{2+}$ transients in cardiac constructs within CNT/Col hydrogels on day 7. (E) Time-course of intracellular $\mathrm{Ca}^{2+}$-transient changes in the CNT/Col group. (F) Average intracellular $\mathrm{Ca}^{2+}$ transients in NRVMs $(\mathrm{n}=26)$ within Col and CNT/Col hydrogels. Data are mean \pm standard error of mean. $* \mathrm{P}<0.05$. All experiments were performed in triplicate.

Abbreviations: CNTs, carbon nanotubes; Col, collagen; NRVMs, neonatal rat ventricular myocytes.

which enhanced cell adhesion and elongation and alignment of cardiomyocytes, leading to the formation of engineered cardiac tissues with stronger contraction potential.

The 3-D nanoscale cues provided by hydrogel matrices have been reported to affect cell adhesion. In our study, CNTs embedded at small concentrations ( $1 \mathrm{wt} \%)$ within Col hydrogels were shown to promote the adhesion of cardiomyocytes remarkably. This result was also supported by a report that mesenchymal stem cells grown in CNT/Col hydrogels exhibited improved cell adhesion and elongation. ${ }^{29}$ A possible explanation for this is that when cardiomyocytes are embedded within the CNT/Col hydrogels, the 3D CNT networks provide sites for the anchorage of cardiomyocytes and direct cytoskeletal extensions. Clearly more studies should explore the mechanisms underlying the facilitative effects of CNTs on cell adhesion.

The structure of myocardial tissue is highly organized in vivo, in which rod-shaped cardiomyocytes arrange in nanoscale cues provided by the extracellular matrix. Nevertheless, when cultured in vitro, cardiomyocytes are deprived of their native organization and follow random distribution on common substrates, thereby blemishing much of their physiology. Therefore, various strategies have been used to direct better-organized cardiomyocytes, including mechanical stretching, electrical stimulation, and substrate topography. ${ }^{8,18,32}$ Although exciting progress, it remains to be explored whether novel nanomaterials exert more facilitative cues for directing cardiomyocytes into anisotropic arrangements in vitro similarly to native myocardium. In the current study, the inclusion of CNTs within Col hydrogels was found to enhance the alignment and integrity of cardiac myocytes. Neonatal cardiomyocytes grown on CNT/Col hydrogels exhibited good subcellular organization and structural alignment similarly to the in vivo ventricular myocardial phenotype, which was supported by immunofluorescent and ultrastructural analysis. After 7 days of culture, cardiomyocytes grown on CNT/Col hydrogels displayed many morphological parameters approaching values previously reported for adult cardiomyocytes in culture, such as cell length, cell area, and sarcomere length. ${ }^{33,34}$

In addition to anisotropic arrangements of cardiomyocytes, another indication for the functional assembly of tissue is the ability to generate synchronous contractions throughout constructed tissues. In this study, CNT/Col hydrogel-based cardiac constructs displayed significantly increased beating frequency 
compared with pure Col hydrogels on day 7. Furthermore, the increase in cell contractility was also supported by calciumtransient measurements. We demonstrated that relatively more synchronous transients and higher magnitudes were noted within CNT/Col hydrogel-based cardiac constructs than in the control group. Such improvement in contractile behavior can account for the enhanced electrophysiological properties. Future work should focus on determining whether topographical cues or the conductivity of CNTs accounts for the enhanced contractile performance of cardiac constructs.

Among the conductive composites used, 2-D CNT-based substrates have been widely studied and shown beneficial effects in cardiomyocyte growth and function. ${ }^{12,13,35}$ Although the results are encouraging, the 2-D nature of conductive substrates impedes their effective application to cardiac regeneration. Clarifying the roles of CNTs will be demanded in the 3-D hydrogel microenvironment, especially for injectable hydrogels. Due to minimally invasive delivery, these hydrogels are favorable for further translation to the treatment of MI. ${ }^{36-38}$ For example, Li et al used poly $(N-$ isopropylacrylamide) hydrogels containing CNTs as carriers of stem cells, which improved cardiac function in rat models of MI. ${ }^{39}$ Here, we incorporated CNTs into Col solution to fabricate CNT/Col hydrogels, which better mimicked native tissue microenvironments and provided more useful information for cardiac tissue engineering. Furthermore, it will be interesting to extend the study of CNT/Col hydrogels to deliver stem cell in vivo for cardiac repair.

\section{Conclusion}

We demonstrated that the inclusion of CNTs within Col hydrogels enhanced cell-cell alignment and assembly of neonatal cardiomyocytes. These cells exhibited anisotropic orientation and synchronous contraction, leading to an increase in tissue-level functionality. The CNT/Col-hydrogel platform should not only provide a useful tool for assessing the impacts of candidate hypertrophic or pharmacologic treatments but also serve as an injectable material to deliver cells or drug molecules for cardiac applications.

\section{Acknowledgments}

This work was supported by the National Natural Science Foundation of China (31400818), a grant from the PLA Medicine Key Project (BWS12J020), and the National Key Clinical Specialist Construction Program of China.

\section{Disclosure}

The authors report no conflicts of interest in this work.

\section{References}

1. Mozaffarian D, Benjamin EJ, Go AS, et al. Heart disease and stroke statistics - 2016 update: a report from the American Heart Association. Circulation. 2016;133:e38-e360.

2. Poole JE, Johnson GW, Hellkamp AS, et al. Prognostic importance of defibrillator shocks in patients with heart failure. N Engl J Med. 2008; 359:1009-1017.

3. Joggerst SJ, Hatzopoulos AK. Stem cell therapy for cardiac repair: benefits and barriers. Expert Rev Mol Med. 2009;11:e20.

4. Hodgkinson CP, Kang MH, Dal-Pra S, Mirotsou M, Dzau VJ. MicroRNAs and cardiac regeneration. Circ Res. 2015;116:1700-1711.

5. Ptaszek LM, Mansour M, Ruskin JN, Chien KR. Towards regenerative therapy for cardiac disease. Lancet. 2012;379:933-942.

6. Hunt JA. Regenerative medicine: materials in a cellular world. Nat Mater. 2008;7:617-618.

7. Langer R, Vacanti JP. Tissue engineering. Science. 1993;260: 920-926.

8. Zimmermann WH, Melnychenko I, Wasmeier G, et al. Engineered heart tissue grafts improve systolic and diastolic function in infarcted rat hearts. Nat Med. 2006;12:452-458.

9. Madden LR, Mortisen DJ, Sussman EM, et al. Proangiogenic scaffolds as functional templates for cardiac tissue engineering. Proc Natl Acad Sci U S A. 2010;107:15211-15216.

10. Chan V, Raman R, Cvetkovic C, Bashir R. Enabling microscale and nanoscale approaches for bioengineered cardiac tissue. ACS Nano. 2013;7:1830-1837.

11. Shin SR, Jung SM, Zalabany M, et al. Carbon-nanotube-embedded hydrogel sheets for engineering cardiac constructs and bioactuators. ACS Nano. 2013;7:2369-2380.

12. Martinelli V, Cellot G, Toma FM, et al. Carbon nanotubes promote growth and spontaneous electrical activity in cultured cardiac myocytes. Nano Lett. 2012;12:1831-1838.

13. Sun H, Lü S, Jiang XX, et al. Carbon nanotubes enhance intercalated disc assembly in cardiac myocytes via the $\beta 1$-integrin-mediated signaling pathway. Biomaterials. 2015;55:84-95.

14. Gaharwar AK, Peppas NA, Khademhosseini A. Nanocomposite hydrogels for biomedical applications. Biotechnol Bioeng. 2014;111: 441-453.

15. Radhakrishnan J, Krishnan UM, Sethuraman S. Hydrogel based injectable scaffolds for cardiac tissue regeneration. Biotechnol Adv. 2014; 32:449-461.

16. Zimmermann WH, Schneiderbanger K, Schubert P, et al. Tissue engineering of a differentiated cardiac muscle construct. Circ Res. 2002;90: $223-230$.

17. de Lange WJ, Hegge LF, Grimes AC, et al. Neonatal mouse-derived engineered cardiac tissue: a novel model system for studying genetic heart disease. Circ Res. 2011;109:8-19.

18. Dvir T, Timko BP, Brigham MD, et al. Nanowired three-dimensional cardiac patches. Nat Nanotechnol. 2011;6:720-725.

19. Tian B, Liu J, Dvir T, et al. Macroporous nanowire nanoelectronic scaffolds for synthetic tissues. Nat Mater. 2012;11:986-994.

20. Tosun Z, McFetridge PS. A composite SWNT-collagen matrix: characterization and preliminary assessment as a conductive peripheral nerve regeneration matrix. J Neural Eng. 2010;7:066002.

21. Koppes AN, Keating KW, McGregor AL, et al. Robust neurite extension following exogenous electrical stimulation within single walled carbon nanotube-composite hydrogels. Acta Biomater. 2016;39:34-43.

22. Dvir T, Levy O, Shachar M, Granot Y, Cohen S. Activation of the ERK1/2 cascade via pulsatile interstitial fluid flow promotes cardiac tissue assembly. Tissue Eng. 2007;13:2185-2193.

23. Tan Y, Richards D, Xu R, et al. Silicon nanowire-induced maturation of cardiomyocytes derived from human induced pluripotent stem cells. Nano Lett. 2015;15:2765-2772.

24. Engelmayr GC, Cheng M, Bettinger CJ, Borenstein JT, Langer R, Freed LE. Accordion-like honeycombs for tissue engineering of cardiac anisotropy. Nat Mater. 2008;7:1003-1010. 
25. Roberts-Thomson KC, Kistler PM, Sanders P, et al. Fractionated atrial electrograms during sinus rhythm: relationship to age, voltage, and conduction velocity. Heart Rhythm. 2009;6:587-591.

26. Liu Z, Davis C, Cai W, He L, Chen X, Dai H. Circulation and long-term fate of functionalized, biocompatible single-walled carbon nanotubes in mice probed by Raman spectroscopy. Proc Natl Acad Sci U S A. 2008; 105:1410-1415.

27. Yang ST, Fernando KA, Liu JH, et al. Covalently PEGylated carbon nanotubes with stealth character in vivo. Small. 2008;4:940-944.

28. MacDonald RA, Voge CM, Kariolis M, Stegemann JP. Carbon nanotubes increase the electrical conductivity of fibroblast-seeded collagen hydrogels. Acta Biomater. 2008;4:1583-1592.

29. Lee JH, Lee JY, Yang SH, Lee EJ, Kim HW. Carbon nanotube-collagen three-dimensional culture of mesenchymal stem cells promotes expression of neural phenotypes and secretion of neurotrophic factors. Acta Biomater. 2014;10:4425-4436.

30. Mao H, Kawazoe N, Chen G. Cellular uptake of single-walled carbon nanotubes in 3D extracellular matrix-mimetic composite collagen hydrogels. J Nanosci Nanotechnol. 2014;14:2487-2492.

31. Chen Y, Bilgen B, Pareta RA, et al. Self-assembled rosette nanotube/ hydrogel composites for cartilage tissue engineering. Tissue Eng Part C Methods. 2010;16:1233-1243.

32. Engelmayr GC, Cheng M, Bettinger CJ, Borenstein JT, Langer R, Freed LE. Accordion-like honeycombs for tissue engineering of cardiac anisotropy. Nat Mater. 2008;7:1003-1010.
33. Olivetti G, Cigola E, Maestri R, et al. Aging, cardiac hypertrophy and ischemic cardiomyopathy do not affect the proportion of mononucleated and multinucleated myocytes in the human heart. J Mol Cell Cardiol. 1996;28:1463-1477.

34. New RB, Zellner JL, Hebbar L, et al. Isolated left ventricular myocyte contractility in patients undergoing cardiac operations. J Thorac Cardiovasc Surg. 1998;116:495-502.

35. Martinelli V, Cellot G, Toma FM, et al. Carbon nanotubes instruct physiological growth and functionally mature syncytia: nongenetic engineering of cardiac myocytes. ACS Nano. 2013;7:5746-5756.

36. Singelyn JM, Sundaramurthy P, Johnson TD, et al. Catheter-deliverable hydrogel derived from decellularized ventricular extracellular matrix increases endogenous cardiomyocytes and preserves cardiac function post-myocardial infarction. J Am Coll Cardiol. 2012;59:751-763.

37. Ungerleider JL, Christman KL. Concise review: injectable biomaterials for the treatment of myocardial infarction and peripheral artery disease - translational challenges and progress. Stem Cells Transl Med. 2014;3:1090-1099.

38. Bidarra SJ, Barrias CC, Granja PL. Injectable alginate hydrogels for cell delivery in tissue engineering. Acta Biomater. 2014;10:1646-1662.

39. Li X, Zhou J, Liu Z, et al. A PNIPAAm-based thermosensitive hydrogel containing SWCNTs for stem cell transplantation in myocardial repair. Biomaterials. 2014;35:5679-5688.
International Journal of Nanomedicine

\section{Publish your work in this journal}

The International Journal of Nanomedicine is an international, peerreviewed journal focusing on the application of nanotechnology in diagnostics, therapeutics, and drug delivery systems throughout the biomedical field. This journal is indexed on PubMed Central, MedLine, CAS, SciSearch ${ }^{\circledR}$, Current Contents ${ }^{\circledR} /$ Clinical Medicine,

\section{Dovepress}

Journal Citation Reports/Science Edition, EMBase, Scopus and the Elsevier Bibliographic databases. The manuscript management system is completely online and includes a very quick and fair peer-review system, which is all easy to use. Visit http://www.dovepress.com/ testimonials.php to read real quotes from published authors. 